\title{
Association of IL-18 polymorphisms with risk of polycystic ovary syndrome in a Han population of China.
}

\author{
Qi Wang*, Zongjian Tan, Hongyun Zhang, Rao Min, Zhang Cheng, Liu Wei \\ Center for Reproductive Medicine, Guizhou Provincial People's Hospital, Guiyang, PR China
}

\begin{abstract}
We aimed to investigate the association of two SNPs in the promoter region of IL-18 (IL-18-607C/A rs1946518 and -137G/C rs187238) with the risk of PCOS in a Chinese Han population. A total of 210 patients with PCOS and 408 subjects without PCOS were selected between June 2015 and December 2016. The genotyping of IL-18-607C/A rs1946518 and -137G/C rs187238 polymorphisms was carried out by an iPlex GOLD SNP genotyping analysis using the Sequenom MassARRAY ${ }^{\circledR}$ System. Unconditional multivariate logistic regression analysis was taken to calculate the relationship of IL-18-607C/A rs1946518 and -137G/C rs187238 polymorphisms with risk of PCOS. We found that BMI $\geq 24$ $(\mathrm{OR}=\mathbf{2 . 7 8}, 95 \% \mathrm{CI}=\mathbf{1 . 8 5}-4.17)$, ever smoking $(\mathrm{OR}=\mathbf{2 . 5 1}, 95 \% \mathrm{CI}=1.08-5.79)$ and ever drinking $(\mathrm{OR}=\mathbf{3 . 6 4}$, 95\% CI=2.33-5.69) were associated with higher risk of PCOS. Those carrying the CC genotype were associated with 2.40 fold risk of developing PCOS $(\mathrm{OR}=\mathbf{2 . 4 0}, 95 \% \mathrm{CI}=\mathbf{1 . 2 2}-4.72)$, and the GC+CC genotype displayed an increased risk of $\operatorname{PCOS}(\mathrm{OR}=2.34,95 \% \mathrm{CI}=1.23-4.48)$. We suggest that IL-18-137G/C could be considered as a predictive factor for the pathogenesis of PCOS in the Han population of China.
\end{abstract}

Keywords: IL-18, -607C/A, -137G/C, Polymorphism, Polycystic ovary syndrome.

Accepted on October 24, 2017

\section{Introduction}

Polycystic ovary syndrome is one of the most common endocrine malfunctions in child-bearing women, which is clinical manifestations of menstrual abnormalities, hair growth, obesity, high blood insulin, and insulin resistance [1]. It is estimated that the incidence of polycystic ovary syndrome is about $7.1 \%-11.2 \%$ in Chinese women aged $12-44$ years [2]. The etiology of polycystic ovary syndrome is very complication and unclear, and many environmental and lifestyle factors greatly contribute to the pathogenesis of the polycystic ovary syndrome [3-6]. However, hereditary factors play a critical role in the pathogenesis of polycystic ovary syndrome. Currently, many studies have reported an association between genetic factors and risk of polycystic ovary syndrome [7-11].

Inflammatory cytokines may be important factors in the pathogenesis of polycystic ovary syndrome. There is convincing evidence describing the influence of low-grade inflammation and cytokines in polycystic ovary syndrome [12-16]. IL-18 is an $18 \mathrm{kDa}$ cytokine, which belongs to the Interleukin-1 (IL-1) superfamily [17]. Two previous studies have indicated a significant association between IL-18 polymorphisms and risk of polycystic ovary syndrome in Asian population $[18,19]$, but they only reported the role of IL-18-237G $>$ C polymorphism in the risk of this disease. Therefore, we aimed to investigate the association of two SNPs in the promoter region of IL-18 (IL-18-607C/A rs1946518 and-137G/C rs187238) with the risk of PCOS in a Chinese Han population.

\section{Methods}

\section{Ethics}

All the investigated respondents voluntary taken part in the study after full understanding of the purpose, and signed informed consent forms before enrolment. The protocol of our study was approved by the ethics committee of Guizhou Provincial People's Hospital.

\section{Patients and controls}

During June 2015 and December 2016, a total of 210 Han patients with PCOS were enrolled from the Center for Reproductive Medicine in Guizhou Provincial People's Hospital. The diagnosis of PCOS was according to the criteria of Rotterdam PCOS consensus in 2003 [20].

Simultaneously, a total of 408 Han healthy individuals without PCOS were randomly selected from the Center for Reproductive Medicine in Guizhou Provincial People's Hospital. Two individuals were matched with one patient with PCOS by age $( \pm 5 \mathrm{y})$. All the controls were diagnosed free of PCOS by $\mathrm{B}$ ultrasonic examination and reproductive hormone 
tests. The exclusion criteria of controls were those with irregular menstrual periods, malignant tumors, autoimmune diseases and ovarian related diseases. The mean ages of patients with PCOS and controls were $28.63 \pm 3.94$ and 27.92 $\pm 4.28 \mathrm{y}$, respectively; the mean age of menarche were $13.07 \pm$ 2.01 and $13.23 \pm 1.86 \mathrm{y}$, respectively; the mean BMI were $22.98 \pm 2.87$ and $21.36 \pm 2.73 \mathrm{~kg} / \mathrm{m}^{2}$, respectively.

Demographic and lifestyle characteristics were collected from the questionnaire survey, including weight, height, alcohol drinking and tobacco smoking habit and physical activity. The Body Mass Index (BMI) is defined as the body mass divided by the square of the body height.

The reproductive hormone indexes were tested by automatic chemiluminescence immune detection system, including Follicle-Stimulating Hormone (FSH), Luteinizing Hormone (LH), Estradiol (E2), Progesterone (P) and Testosterone (T).

\section{Genotyping}

Five $\mathrm{ml}$ of peripheral venous blood were obtained from each respondent. The blood samples were centrifuged to separate serum, and stored at $-80^{\circ} \mathrm{C}$ until use. Genomic DNA was isolated from peripheral blood samples with Blood DNA extraction kit. The DNA samples were kept at $-20^{\circ} \mathrm{C}$ until using. The genotyping of IL-18-607C/A rs1946518 and-137G/C rs187238 polymorphisms was carried out by an iPlex GOLD SNP genotyping analysis using the Sequenom MassARRAY ${ }^{\circledR}$ System (Sequenom, San Diego, USA). The primers used for amplification of IL-18-607C/A and-137G/C were designed by Sequenom Assay Design 3.1 software.

Then the SAP and iPLEX reactions were carried out to analyse the PCR amplification products.

The PCR products are then desalted and crystallized, and then analysed by SpectroCHIP and MALDI-TOF MS reaction. The mass spectra peak and genotypes of IL-18-607C/A and-137G/C was analysed by Typer 4.0 software.

\section{Statistical analysis}

Continuous variables were displayed as means \pm Standard Deviations (SD), and categorical variables were shown as percentages and frequencies (\%). Comparison of demographic, lifestyle and clinical characteristics between the PCOS patients and controls were calculated using Chi-square $\left(\chi^{2}\right)$ test or student t test. Chi-square $\left(\chi^{2}\right)$ test with one degree of freedom was used to estimate whether the IL-18-607C/A rs1946518 and-137G/C rs 187238 genetic frequencies were in line with the Hardy-Weinberg equilibrium. Unconditional multivariate logistic regression analysis was taken to calculate the relationship of IL-18-607C/A rs1946518 and-137G/C rs187238 polymorphisms with risk of PCOS. Odds Ratios (ORs) and 95\% Confidence Intervals (CIs) were used to express the results. Interaction between environmental factors and genetic polymorphisms of IL-18-607C/A rs1946518 and-137G/C rs187238 was estimated by spearman correlation analysis. All the data were analysed with the software IBM
SPSS Statistics for Windows, Version 20.0. (IBM Corp., Armonk, NY, USA). Two tailed $\mathrm{P}<0.05$ was regarded as statistical significant difference.

\section{Results}

Comparison with controls, patients with PCOS were more likely to have an early age of menarche, high level of BMI, FSH, LH and T, low level of P, and a habit of smoking and drinking (Table 1).

The genotype distributions of the IL-18-607C/A and-137G/C were according to the $\mathrm{HWE}$ in both patients (for-607C/A: $\chi^{2}=2.42, \mathrm{P}=0.09$; for-137G/C: $\chi^{2}=0.86, \mathrm{P}=0.35$ ) and controls (for-607C/A: $\chi^{2}=1.99, \mathrm{P}=0.16$; for-137G/C: $\chi^{2}=3.12, \mathrm{P}=0.07$ ) (Table 2).

By multivariate logistic regression analysis, we found that BMI $\geq 24(\mathrm{OR}=2.78,95 \% \mathrm{CI}=1.85-4.17)$, ever smoking $(\mathrm{OR}=2.51$, $95 \% \quad \mathrm{CI}=1.08-5.79)$ and ever drinking (OR=3.64, 95\% $\mathrm{CI}=2.33-5.69)$ were associated with higher risk of PCOS when compared with the reference group (Table 3). Compared with individuals carrying GG genotype of IL-18-137G/C, those carrying the $\mathrm{CC}$ genotype were associated with 2.40 fold risk of developing PCOS $(\mathrm{OR}=2.40,95 \% \mathrm{CI}=1.22-4.72)$, and the $\mathrm{GC}+\mathrm{CC}$ genotype displayed an increased risk of PCOS $(\mathrm{OR}=2.34,95 \% \mathrm{CI}=1.23-4.48)$.

We found that IL-18-137G/C polymorphisms was associated with $\mathrm{BMI} \geq 24$ in the risk of PCOS, while the IL-18-137G/C polymorphism had no interaction with, age, age of menarche, smoking and drinking in the risk of PCOS (Table 4). Moreover, we observed that IL-18-137G/C polymorphism had no interaction with $\mathrm{FSH}, \mathrm{LH}, \mathrm{E} 2, \mathrm{P}$ and $\mathrm{T}$ levels in the risk of PCOS (Table 5).

Table 1. Demographic and clinical characteristics of patients with PCOS and controls.

\begin{tabular}{lllll}
\hline \multirow{2}{*}{ Variables } & Patients (\%) & Controls (\%) & \multirow{2}{*}{$\mathbf{X}^{2}$ or t value } & P value \\
\cline { 2 - 3 } & $\mathbf{n = 2 1 0}$ & $\mathbf{n = 4 0 8}$ & \\
\hline Age, years & $28.63 \pm 3.94$ & $27.92 \pm 4.28$ & -1.01 & 0.32 \\
\hline $\begin{array}{lllll}\text { Age of menarche, } \\
\text { years }\end{array}$ & $13.07 \pm 2.01$ & $13.23 \pm 1.86$ & 2.01 & 0.045 \\
\hline Weight, kg & $58.38 \pm 5.92$ & $54.94 \pm 5.57$ & 7.13 & $<0.001$ \\
\hline Height, m & $1.60 \pm 0.06$ & $1.61 \pm 0.06$ & -1.82 & 0.07 \\
\hline BMI, kg/m 2 & $22.98 \pm 2.87$ & $21.36 \pm 2.73$ & 6.84 & $<0.001$ \\
\hline$\geq 24$ & $71(33.81)$ & $65(15.93)$ & 25.82 & $<0.001$ \\
\hline Ever smoking & $14(6.67)$ & $11(2.94)$ & 4.78 & 0.03 \\
\hline Ever drinking & $65(30.00)$ & $72(12.01)$ & 30.24 & $<0.001$ \\
\hline FSH & $4.47 \pm 2.28$ & $3.68 \pm 2.15$ & 4.25 & $<0.001$ \\
\hline LH & $14.83 \pm 4.54$ & $11.33 \pm 3.21$ & 11.11 & $<0.001$ \\
\hline E2 & $91.93 \pm 21.21$ & $91.10 \pm 21.77$ & 0.45 & 0.65 \\
\hline P & $7.26 \pm 2.92$ & $8.11 \pm 3.05$ & -3.35 & 0.001 \\
\hline
\end{tabular}


Association of IL-18 polymorphisms with risk of polycystic ovary syndrome in a Han population of China

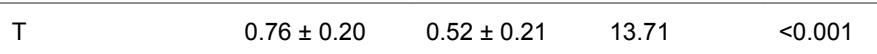

Table 2. Genotype distributions of IL-18 -607C/A rs 1946518 and $-137 \mathrm{G} / \mathrm{C}$ rs 187238 between the two study groups.

\begin{tabular}{|c|c|c|c|c|c|c|c|c|c|c|c|c|}
\hline \multirow[t]{2}{*}{ IL-18 } & \multicolumn{3}{|c|}{ Patients (\%) } & \multirow[t]{2}{*}{ Controls (\%) } & \multirow[t]{2}{*}{$x^{2}$ test } & \multirow[t]{2}{*}{$P$ value } & \multicolumn{3}{|c|}{ HWE in patients } & \multicolumn{3}{|c|}{ HWE in controls } \\
\hline & & & & & & & $x^{2}$ & \multicolumn{2}{|c|}{$P$ value } & $x^{2}$ & \multicolumn{2}{|c|}{$P$ value } \\
\hline \multicolumn{13}{|l|}{$-607 \mathrm{C} / \mathrm{A}$} \\
\hline $\mathrm{CC}$ & \multicolumn{3}{|c|}{$73(34.76)$} & $135(33.09)$ & & & & & & & & \\
\hline $\mathrm{CA}$ & \multicolumn{3}{|c|}{$87(41.43)$} & $187(45.83)$ & & & & & & & & \\
\hline AA & \multicolumn{3}{|c|}{$50(23.81)$} & $86(21.08)$ & 1.19 & 0.55 & 2.42 & \multicolumn{2}{|c|}{0.09} & 1.99 & \multicolumn{2}{|l|}{0.16} \\
\hline \multicolumn{13}{|l|}{$-137 \mathrm{G} / \mathrm{C}$} \\
\hline GG & \multicolumn{3}{|c|}{$89(42.38)$} & $194(47.55)$ & & & & & & & & \\
\hline GC & \multicolumn{3}{|c|}{$100(47.62)$} & $191(46.81)$ & & & & & & & & \\
\hline $\mathrm{CC}$ & \multicolumn{3}{|c|}{$21(10.00)$} & $23(5.64)$ & 4.53 & 0.1 & 0.86 & \multicolumn{2}{|c|}{0.35} & 3.12 & \multicolumn{2}{|l|}{0.07} \\
\hline \multirow{2}{*}{\multicolumn{6}{|c|}{$\begin{array}{l}\text { Table 3. Unconditional multivariate logistic regression analysis for } \\
\text { the risk factors of PCOS. }\end{array}$}} & $\mathrm{CA}+\mathrm{AA}$ & -0.075 & 0.179 & 0.174 & \multirow{2}{*}{\multicolumn{2}{|c|}{$0.93(0.65-1.32)$}} & \multirow[t]{2}{*}{0.68} \\
\hline & & & & & & \multicolumn{4}{|l|}{$-137 \mathrm{G} / \mathrm{C}$} & & & \\
\hline Variables & $\beta$ & S.E. & Wals & Adjusted OR (95\% Cl) & P value & GG & & & & 1.0 (Ref.) & & \\
\hline \multicolumn{6}{|l|}{$-607 \mathrm{C} / \mathrm{A}$} & GC & 0.04 & 0.19 & 0.04 & \multicolumn{2}{|l|}{$1.04(0.72-1.50)$} & 0.85 \\
\hline \multicolumn{4}{|l|}{$\mathrm{CC}$} & \multicolumn{2}{|l|}{1.0 (Ref.) } & $\mathrm{CC}$ & 0.88 & 0.35 & 5.46 & \multicolumn{2}{|l|}{$2.40(1.22-4.72)$} & 0.01 \\
\hline $\mathrm{CA}$ & -0.15 & 0.19 & 0.6 & $0.86(0.59-1.26)$ & 0.44 & $\mathrm{GC}+\mathrm{CC}$ & 0.85 & 0.33 & 6.64 & $2.34(1.23-4.48)$ & & 0.01 \\
\hline AA & 0.07 & 0.23 & 0.1 & 1.08 (0.69-1.69) & 0.75 & & & & & & & \\
\hline
\end{tabular}

Table 4. Interaction between environmental factors and IL-18-137G/C in the risk of PCOS.

\begin{tabular}{|c|c|c|c|c|c|c|c|c|}
\hline \multirow[t]{2}{*}{ Variables } & \multicolumn{2}{|l|}{ Patients } & \multirow{2}{*}{$\begin{array}{l}\mathrm{x}^{2} \text { or } \mathbf{t} \\
\mathbf{G G}\end{array}$} & \multirow[t]{2}{*}{$P$ value } & \multirow{2}{*}{\multicolumn{2}{|c|}{$\begin{array}{l}\text { Controls } \\
\text { GC+CC }\end{array}$}} & \multirow[t]{2}{*}{$\mathrm{x}^{2}$ or $\mathrm{t}$} & \multirow[t]{2}{*}{$P$ value } \\
\hline & GG & $\mathrm{GC}+\mathrm{CC}$ & & & & & & \\
\hline Age $(y)$ & $28.63 \pm 4.00$ & $28.66 \pm 3.53$ & -0.04 & 0.97 & $27.97 \pm 4.28$ & $27.16 \pm 4.14$ & 0.88 & 0.38 \\
\hline Age of menarche, years & $13.04 \pm 2.13$ & $13.33 \pm 1.27$ & -0.64 & 0.52 & $13.21 \pm 1.87$ & $13.51 \pm 1.74$ & -0.74 & 0.46 \\
\hline \multicolumn{9}{|l|}{ BMI } \\
\hline$<24$ & 120 & 19 & & & 325 & 18 & & \\
\hline$\geq 24$ & 69 & 2 & 0.61 & 0.43 & 60 & 5 & 6.15 & 0.01 \\
\hline \multicolumn{9}{|l|}{ Smoking } \\
\hline Never & 176 & 20 & & & 374 & 22 & & \\
\hline Ever & 13 & 1 & 0.17 & 0.68 & 11 & 1 & 0.14 & 0.71 \\
\hline \multicolumn{9}{|l|}{ Drinking } \\
\hline Never & 130 & 17 & & & 338 & 21 & & \\
\hline Ever & 59 & 4 & 0.25 & 0.62 & 47 & 2 & 1.33 & 0.25 \\
\hline
\end{tabular}

Table 5. Interaction between clinical factors and IL-18-137G/C in the risk of PCOS.

\begin{tabular}{llllll}
\hline Variables & Patients & & P value & Controls & P value \\
\cline { 2 - 3 } & GG & GC+CC & GG & GC+CC \\
\hline
\end{tabular}




\begin{tabular}{lllllllll}
\hline FSH & $4.40 \pm 2.23$ & $5.11 \pm 2.67$ & -1.36 & 0.177 & $3.72 \pm 2.16$ & $3.03 \pm 1.95$ & 1.49 & 0.14 \\
\hline LH & $14.90 \pm 4.54$ & $14.22 \pm 4.57$ & 0.65 & 0.514 & $11.35 \pm 3.23$ & $10.94 \pm 2.89$ & 0.6 & 0.55 \\
\hline E2 & $91.80 \pm 20.97$ & $93.10 \pm 23.78$ & -0.27 & 0.79 & $90.53 \pm 21.76$ & $100.53 \pm 20.14$ & -2.05 & 0.06 \\
\hline P & $7.23 \pm 2.93$ & $7.54 \pm 2.88$ & -0.47 & 0.641 & $8.12 \pm 2.99$ & $8.04 \pm 3.95$ & 0.11 & 0.91 \\
\hline T & $0.77 \pm 0.20$ & $0.74 \pm 0.24$ & 0.49 & 0.622 & $0.52 \pm 0.21$ & $0.52 \pm 0.15$ & 0.08 & 0.94 \\
\hline
\end{tabular}

\section{Discussion}

In the present study, we found that IL-18-607C/A polymorphism was significant associated with an increased risk of PCOS. Chronic low-grade inflammation is reported to be correlated with abnormal metabolism in PCOS patients, and it is resulted from abnormal endometrium implantation $[15,16]$. Liu et al. performed a prospective study on the association between serum levels of TSP-1, NF- $\kappa$ B and TGF- $\beta 1$ and PCOS, and they found that the testosterone, Free Androgen Index (FAI), Luteinizing Hormone/Follicle-Stimulating Hormone (LH/FSH) ratio, HOMA-IR, TSP-1 and NF- $\kappa$ B were significantly higher in the PCOS groups than those in the control group [21]. Yang et al. performed a study on the association of serum IL-18 concentrations and PCOS, and they found that IL-18 level was increased in PCOS patients and correlated with insulin resistance, obesity and hyperandrogenism [22].

Three previous studies investigated the association between IL-18 polymorphisms and risk of polycystic ovary syndrome $[18,19,23]$. Yang et al. performed a study with 118 Chinese women with PCOS and 79 controls, and they found a significant different in the $\mathrm{G}$ and $\mathrm{C}$ allele frequencies of IL-18-137G/C between the two study groups, and those carrying the $\mathrm{C}$ allele of $-137 \mathrm{G} / \mathrm{C}$ may contribute to the risk of PCOS in a Chinese population [18]. Kim et al. performed a study in a Korean population with 126 women with PCOS and 113 controls, and they reported that the IL-18-137G allele could serve an important role in the predisposition to glucose intolerance in Korean women with PCOS [18]. Deligeoroglou et al. performed study in Greeks, and they found that IL-18 is elevated in lean patients and raised in the presence of obesity and insulin resistance [23]. However, some studies reported inconsistent results. Wu et al. performed a meta-analysis with eighteen studies, and they found no association between IL-18-607C/A and-137G/C polymorphisms and risk of PCOS [24]. In our study, we found that IL-18-137G/C polymorphism was associated with PCOS, while no correlation between IL-18-607C/A and this disease. Therefore, further studies are greatly needed to confirm our findings.

There are three limitations should be mentioned in the present study. First, since patients and controls were only enrolled from one place of China, these participants may not well represent individuals in other places, and the selection bias may be inevitable. Second, the sample size of patients and controls was relatively small, which may result in a low statistical power to identify differences between groups. Third, this study is a case-control study design, and the recall bias may influence the reliability of data.

In conclusion, this study provides a new insight in PCOS that IL-18-137G/C genotype is associated with the risk of PCOS. We suggest that IL-18-137G/C could be considered as a predictive factor for the pathogenesis of PCOS in the Han population of China.

\section{Acknowledgements}

We thank great help from staffs in Guizhou Provincial People's Hospital, and they help us to collect the blood samples from enrolled subjects.

\section{References}

1. Legro RS, Castracane VD, Kauffman RP. Detecting insulin resistance in polycystic ovary syndrome: purposes and pitfalls. Obstet Gynecol Surv 2004; 59: 141-154.

2. Zhuang J, Liu Y, Xu L, Liu X, Zhou L, Tang L, Kang D, Guo W, He M, Yang F, Qiu D. Prevalence of the polycystic ovary syndrome in female residents of Chengdu, China. Gynecol Obstet Invest 2014; 77: 217-223.

3. Piltonen TT. Polycystic ovary syndrome: Endometrial markers. Best Pract Res Clin Obstet Gynaecol 2016; 37: 66-79.

4. Merkin SS, Phy JL, Sites CK, Yang D. Environmental determinants of polycystic ovary syndrome. Fertil Steril 2016; 106: 16-24.

5. Unluturk U, Sezgin E, Yildiz BO. Evolutionary determinants of polycystic ovary syndrome: part 1. Fertil Steril 2016; 106: 33-41.

6. Fessler DMT, Natterson-Horowitz B, Azziz R. Evolutionary determinants of polycystic ovary syndrome: part 2. Fertil Steril 2016; 106: 42-47.

7. Ben Salem A, Megdich F, Kacem O, Souayeh M, Hachani Ben Ali F, Hizem S, Janhai F, Ajina M, Abu-Elmagd M, Assidi M, Al Qahtani MH, Mahjoub T. Vascular endothelial growth factor (VEGFA) gene variation in polycystic ovary syndrome in a Tunisian women population. BMC Genomics 2016; 17: 748.

8. Banerjee U, Dasgupta A, Khan A, Ghosh MK, Roy P, Rout JK, Roy P, Dhara S. A cross-sectional study to assess any possible linkage of $\mathrm{C} / \mathrm{T}$ polymorphism in CYP17A1 gene with insulin resistance in non-obese women with polycystic ovarian syndrome. Indian J Med Res 2016; 143: 739-747.

9. Talaat RM, Mohamed YA, Mohamad EH, Elsharkawy M, Guirgis AA. Interleukin 10 (- 1082 G/A) and (- 819 C/T) 
gene polymorphisms in Egyptian women with polycystic ovary syndrome (PCOS). Meta Gene 2016; 9: 254-258.

10. Tang W, Wang Y, Jiang H, Liu C, Dong C, Chen S, Kang $\mathrm{M}, \mathrm{Gu} \mathrm{H}$. Insulin receptor substrate-1 (IRS-1) rs $1801278 \mathrm{G}>\mathrm{A}$ polymorphism is associated with polycystic ovary syndrome susceptibility: a meta-analysis. Int J Clin Exp Med 2015; 8: 17451-17460.

11. Gammoh E, Mahmood NA, Madan S, Ebrahim BH, Mustafa FE, Almawi WY. Transcription factor-7-like 2 gene variants affect the metabolic phenotypes of polycystic ovary syndrome. Ann Nutr Metab 2015; 67: 228-235.

12. Palomba S, Falbo A, Chiossi G, Orio F, Tolino A, Colao A, La Sala GB, Zullo F. Low-grade chronic inflammation in pregnant women with polycystic ovary syndrome: a prospective controlled clinical study. J Clin Endocrinol Metab 2014; 99: 2942-2951.

13. Spritzer PM, Lecke SB, Satler F, Morsch DM. Adipose tissue dysfunction, adipokines, and low-grade chronic inflammation in polycystic ovary syndrome. Reproduction 2015; 149: 219-227.

14. Repaci A, Gambineri A, Pasquali R. The role of low-grade inflammation in the polycystic ovary syndrome. Mol Cell Endocrinol 2011; 335: 30-41.

15. Riley JK, Jungheim ES. Is there a role for diet in ameliorating the reproductive sequelae associated with chronic low-grade inflammation in polycystic ovary syndrome and obesity? Fertil Steril 2016; 106: 520-527.

16. Shorakae S, Teede H, de Courten B, Lambert G, Boyle J and Moran LJ. The emerging role of chronic low-grade inflammation in the pathophysiology of polycystic ovary syndrome. Semin Reprod Med 2015; 33: 257-269.

17. Dinarello CA. IL-18: A TH1-inducing, proinflammatory cytokine and new member of the IL-1 family. J Allergy Clin Immunol 1999; 103: 11-24.

18. Yang Y, Qiao J, Li MZ. Association of polymorphisms of interleukin-18 gene promoter region with polycystic ovary syndrome in Chinese population. Reprod Biol Endocrinol 2010; 8: 125.

19. Kim JW, Lee MH, Park JE, Yoon TK, Lee WS, Shim SH. Association of IL-18 genotype with impaired glucose regulation in Korean women with polycystic ovary syndrome. Eur J Obstet Gynecol Reprod Biol 2012; 161: 51-55.

20. Rotterdam ESHRE/ASRM-Sponsored PCOS Consensus Workshop Group. Revised 2003 consensus on diagnostic criteria and long-term health risks related to polycystic ovary syndrome (PCOS). Hum Reprod 2004; 19: 41-47.

21. Liu M, Gao J, Zhang Y, Li P, Wang H, Ren X, Li C. Serum levels of TSP-1, NF-?B and TGF- $\beta 1$ in polycystic ovarian syndrome (PCOS) patients in northern China suggest PCOS is associated with chronic inflammation. Clin Endocrinol (Oxf) 2015; 83: 913-922.

22. Yang Y, Qiao J, Li R, Li MZ. Is interleukin-18 associated with polycystic ovary syndrome? Reprod Biol Endocrinol 2011; 9: 7.

23. Deligeoroglou E, Vrachnis N, Athanasopoulos $\mathrm{N}$, Iliodromiti Z, Sifakis S, Iliodromiti S, Siristatidis C, Creatsas G. Mediators of chronic inflammation in polycystic ovarian syndrome. Gynecol Endocrinol 2012; 28: 974-978.

24. Wu H, Yu K, Yang Z. Associations between TNF- $\alpha$ and interleukin gene polymorphisms with polycystic ovary syndrome risk: a systematic review and meta-analysis. J Assist Reprod Genet 2015; 32: 625-634.

\section{*Correspondence to}

Qi Wang

Center for Reproductive Medicine

Guizhou Provincial People's Hospital

PR China 